\title{
Prevalence of symptoms at the end of life in an acute care hospital: a retrospective cohort study
}

\author{
Daniel Kobewka MD MSc, Paul Ronksley PhD, Dan McIsaac MD MPH, Sunita Mulpuru MD MSc, \\ Alan Forster MD MSc
}

Abstract

Background: There is currently debate over the benefits and harms of physician-assisted death. One of the factors influencing this debate is concern about symptoms in the days before death. The objective of this study was to describe the frequency of symptoms before death and determine patient characteristics associated with these symptoms.

Methods: We reviewed the medical record of every patient who died at a multisite academic teaching hospital over a 3-month period. We determined the number of episodes of pain, dyspnea, agitation and nausea during the final 48 hours of life and assessed the patient and encounter characteristics associated with 2 or more episodes of symptoms.

Results: A total of 480 patients died during the study period. Of these patients, $29.2 \%(140 / 480)$ had 2 or more symptoms in the final 48 hours of life. Higher Elixhauser comorbidity scores (relative risk [RR] 1.35, 95\% confidence interval [Cl] 1.23-1.49), having a family doctor (RR 2.33,95\% Cl 1.02-5.38), being admitted to the medical oncology service (RR 1.51, 95\% Cl 1.11-2.05) and having a documented order for no resuscitation written early during the stay in hospital (RR 1.38, 95\% $\mathrm{Cl} 1.01-1.89$ ) were independently associated with symptoms. Admission to intensive care was associated with fewer symptoms (RR 0.39, CI 95\% 0.19-0.80).

Interpretation: Symptoms are common in the final 48 hours of life, particularly in patients with multimorbidity who want limitations on the aggressiveness of their care. An integrated palliative approach is needed for select at-risk patients.

T he care of terminally ill patients has become a substantial issue facing providers and policy-makers. Heightening this attention are the evolving perspectives on physician-assisted death by various patient advocacy groups and the general public. It is likely these views are driven in part by recognition that many patients experience symptoms at the time of death. Avoiding symptoms is important to patients at the end of life and is likely a primary motivation for physician-assisted death. ${ }^{1-3}$

Our knowledge of terminal patients' experiences is limited. In Canada, as in many countries, most deaths occur in acute care hospitals. 4,5 This may not be the most appropriate location to provide end-of-life care, ${ }^{6}$ but it does create an opportunity to evaluate symptoms in a large proportion of dying patients. Previous research is dated or has focused mostly on disease-specific cohorts. ${ }^{7-11}$ These studies, although limited in focus, do suggest that a substantial proportion of patients have poor symptom control at the time of death. ${ }^{12-14}$

We performed this study to describe the epidemiology of symptoms before death for patients in a multisite academic health sciences centre. This information will guide better ser- vice delivery by instructing providers on the extent of any potential care gap between patient desires and outcomes. More importantly, it will help define target populations to maximize the impact of any interventions, such as enhanced palliative care services and physician-assisted death.

\section{Methods}

\section{Study design and population}

We conducted this study at The Ottawa Hospital - an academic health sciences centre with 3 campuses, 1065 beds and a catchment area population of about 1 million. We included all adult inpatients who died in hospital between Sept. 5 and

Competing interests: None declared.

This article has been peer reviewed.

Correspondence to: Daniel Kobewka, dkobewka@gmail.com

CMAJ Open 2017. DOI:10.9778/cmajo.20160123 
Dec. 16, 2013. The study period was a convenience sample for which baseline data had already been collected as part of a related study. We excluded people who died in the emergency department before admission.

\section{Data sources}

Data were extracted from 2 main sources, the Ottawa Hospital Data Warehouse (OHDW), and from direct review of the patient's medical record by a clinician. The OHDW is a relational database that contains clinical and administrative information extracted from the hospital's information systems, including the patient registration system, clinical data repository and patient abstracts. Professional coders use International Statistical Classification of Diseases and Related Health Problems, 10th revision, Canadian version, to enter diagnostic codes.

\section{Study variables}

Using a standardized chart extraction form, a clinician reviewed the medical record from the final hospital admission of each patient. The form was initially piloted on 10 charts to ensure usability and feasibility for collecting each data element. Minor modifications were made extracting data for the full cohort. Information on patient decision-making about resuscitation was collected to understand the wishes of people with more symptoms at the end of life. In addition, early documentation of patient preferences for resuscitation is a quality indicator for end-of-life care. ${ }^{15,16}$ The variables extracted from the patient's medical record included the patient's wishes for cardiopulmonary resuscitation (CPR) and mechanical ventilation as documented during the first 24 hours of admission, any order to withhold CPR and mechanical ventilation written before the time of death, the number of days before death the resuscitation orders were written, whether family was present at any point in the final 24 hours of life, and symptoms of pain, dyspnea, agitation or nausea occurring in the final 48 hours of life.

Using the OHDW, we extracted information regarding patient demographics, previous inpatient encounters, total length of hospital stay before death, whether the patient had a family physician, hospital service on admission and at the time of death, most responsible diagnosis that caused the patients' hospital stay, Elixhauser comorbidities, Elixhauser score and baseline risk of death at time of admission. ${ }^{17,18}$ The Elixhauser comorbidity score is calculated from 30 different medical conditions identified through administrative data. The score summarizes disease burden and is correlated with the risk of in-hospital death. The estimated baseline risk of death in hospital was calculated using a validated risk score that uses laboratory test values, patient demographics and comorbidities. ${ }^{19}$

\section{Outcomes}

The primary outcome was the incidence of symptoms during the final 48 hours of life, as documented in the patient chart. We counted each documented episode of pain, dyspnea, nausea or agitation. These symptoms were selected because they are common at the end of life and cause considerable distress for many patients. ${ }^{1,20}$ When pain was documented with a rating on a scale anchored at 0 (no pain) and 10 (worst possible pain), we only counted pain scoring 5 or higher. We used this threshold to capture pain that was likely causing distress and not simply a nuisance. ${ }^{21-23}$ During the study period, our hospital had an hourly rounding policy that required nurses to assess each patient every hour. There was, however, no standardized treatment pathway to deal with symptoms.

\section{Analysis}

We described the frequency of each symptom in the final 48 hours of life. We used means ( \pm standard deviation [SD]), medians (interquartile range $[\mathrm{IQR}]$ ) and proportions as appropriate to describe the characteristics of patients who died during the study period. We compared characteristics of patients who had 2 or more episodes of symptoms in the final 48 hours of life with those who had fewer than 2 episodes of symptoms in the final 48 hours of life. We chose a cut-off of 2 symptomatic episodes because patients with multiple symptomatic episodes are more likely to have a poor-quality death and have more to gain from symptom-reducing interventions. ${ }^{24} \mathrm{We}$ used $\chi^{2}$ or 2 -sample $t$-tests as appropriate for the bivariate analysis.

We developed multivariable logarithmic binomial regression models to identify patient and admission characteristics associated with the presence of 2 or more symptoms in the final 48 hours of life. We chose logarithmic binomial regression because of the intuitive interpretation of relative risks (RRs) and the divergence of odds ratios and RRs when an outcome is common..$^{25,26} \mathrm{~A}$ stepwise model-building approach was selected using RRs with corresponding 95\% confidence intervals (CIs) as the common measure of association. All patient and admission characteristics that had $p$ values of 0.10 or below in the bivariate analysis were used for multivariate analysis. Specifically, we tested patient characteristics (age, sex, Elixhauser comorbidity score at admission [modelled as a continuous variable] and presence of a family physician), hospital admission characteristics (admitting service, number of prior admissions in past $6 \mathrm{mo}$ and involvement of a palliative care physician) and patient wishes regarding resuscitation and mechanical ventilation at the time of admission. Variables that had a potential for reverse causation given their timing related to the outcome of interest were not included in multivariate analysis. This included patients' wishes for resuscitation recorded at the time of death and presence of family during the final 24 hours of life. We then used backward elimination techniques $(\alpha=0.05)$ to develop reduced models. All analyses were completed using SAS version 9.3 statistical software.

To further describe patients who died with symptoms we selected several representative cases to describe in narrative format. These cases were pseudonymized to maintain confidentiality.

\section{Ethics approval}

This study was approved by the Ottawa Health Science Network Research Ethics Board. 


\section{OPEN}

Research

\section{Results}

During the study period, 14266 patients were admitted to hospital. The mean age of patients was 48.9 ( \pm 27.3 ) years, $56.5 \%$ were female, and the median length of stay was 3 (IQR $2-7)$ days. There were 480 deaths during the study period (3.4\% mortality). Among decedents, the mean age was 73.7 ( \pm 16.1) years, $47.5 \%$ were female, and the median length of stay was 7 (IQR 3-17) days. The medical record of 1 patient could not be located and was therefore excluded from further analysis.

The most common comorbidities in the population are presented in Table 1 . The 5 most common most responsible diagnoses were palliative care $(21.3 \%)$, sepsis (5.2\%), congestive heart failure $(4.8 \%)$, pneumonia $(2.5 \%)$ and exacerbation of chronic obstructive pulmonary disease (2.1\%).

Among the 479 patients who died and had complete records, 207 (43.2\%) had at least 1 symptom documented in the final 48 hours of life, and 140 (29.2\%) had at least 2. Pain and dyspnea were the most common symptoms, while nausea was the least common (Table 2).

\begin{tabular}{|lc|}
\hline $\begin{array}{l}\text { Table 1: The } 10 \text { most common comorbidities in the decedent } \\
\text { cohort }\end{array}$ & $\begin{array}{c}\text { Prevalence, no. (\%) } \\
n=479\end{array}$ \\
\hline Elixhauser comorbidity & $196(40.8)$ \\
\hline Hypertension & $157(32.7)$ \\
\hline Cancer with no metastases & $145(30.2)$ \\
\hline Arrythmia & $120(25.0)$ \\
\hline Diabetes with complications & $113(23.5)$ \\
\hline Cancer with metastases & $105(21.9)$ \\
\hline Chronic heart failure & $99(20.6)$ \\
\hline Fluid and electrolyte disorders & $77(16.0)$ \\
\hline Chronic obstructive pulmonary disease & $75(15.6)$ \\
\hline Diabetes without complications & $61(12.7)$ \\
\hline Chronic kidney disease & \\
\hline
\end{tabular}

Table 3 shows the characteristics of patients with 2 or more episodes of symptoms in the final 48 hours of life compared with those with fewer than 2 episodes. Patients with 2 or more episodes of symptoms in the final 48 hours of life had significantly more inpatient encounters in the preceding 6 months, more inpatient days in the last year, longer length of stay and higher Elixhauser comorbidity scores compared with patients who had fewer than 2 episodes (Table 3).

Patients with 2 or more episodes of symptoms were also more likely to have a family physician, more likely to be admitted to an oncology service and less likely to be admitted to the intensive care unit (ICU). Patients with 2 or more episodes of symptoms were more likely to have a wish for "no resuscitation, no ICU admission" documented within 24 hours of admission (47.1\% v. 34.4\%, $p=0.01$ ), compared with those with fewer than 2 episodes. Patients with symptoms before death were also more likely to have a no resuscitation order documented in the chart at the time of death $(90.7 \% \mathrm{v}$. $71.1 \%$ ), were less likely to have this order written within 24 hours of death (12.9\% v. $23.9 \%)$ and were more likely to be seen by a palliative care physician before death $(45.0 \% \mathrm{v}$. $23.8 \%)$. It is notable that there were no differences in age or the predicted risk of death on admission for patients with or without symptoms before death.

Within our fully adjusted multivariable regression model, we found that higher Elixhauser score (RR 1.35, 95\% CI 1.23-1.49), having a family doctor (RR 2.33, 95\% CI $1.02-$ 5.38 ), being admitted to medical oncology (RR 1.51, 95\% CI 1.11-2.05) and having a documented wish for no CPR and no intubation during the first 24 hours in hospital (RR 1.38, 95\% CI 1.01-1.89) were all associated with symptoms at the end of life. Being admitted to the ICU was associated with fewer symptoms at the end of life (RR 0.39 , 95\% CI 0.19-0.80) (Figure 1). Table 4 contains 3 representative case vignettes of patients who died with more than 2 episodes of symptoms.

\section{Discussion}

We found that almost 1 in 3 people who died in hospital had 2 or more episodes of symptoms in the final 48 hours of life.

\begin{tabular}{|c|c|c|c|c|c|}
\hline \multirow[b]{2}{*}{$\begin{array}{l}\text { Frequency of } \\
\text { symptom(s) }\end{array}$} & \multicolumn{5}{|c|}{$\begin{array}{l}\text { No. (\%) of patients with each symptom } \\
\qquad n=479\end{array}$} \\
\hline & Pain & Dyspnea & Agitation & Nausea & $\begin{array}{l}\text { Total with any } \\
\text { symptom(s) }\end{array}$ \\
\hline 0 & 376 (78.5) & 373 (77.9) & 402 (83.9) & $459(95.8)$ & $272(56.8)$ \\
\hline 1 & $49(10.2)$ & $54(11.3)$ & $45(9.4)$ & $12(2.5)$ & $67(14.0)$ \\
\hline 2 & $27(5.6)$ & 32 (6.7) & $14(2.9)$ & $6(2.5)$ & $45(9.4)$ \\
\hline 3 & $15(3.1)$ & $13(2.7)$ & $10(2.1)$ & $1(1.3)$ & $44(9.2)$ \\
\hline 4 & $8(1.7)$ & $4(0.8)$ & $5(1.0)$ & $0(0)$ & 18 (3.8) \\
\hline$\geq 5$ & $4(0.8)$ & $3(0.6)$ & $3(0.6)$ & $1(0.6)$ & $33(6.9)$ \\
\hline
\end{tabular}




\begin{tabular}{|c|c|c|c|}
\hline Characteristic & $\begin{array}{l}\geq 2 \text { episodes of distressing } \\
\text { symptoms before death, no. (\%)* } \\
\qquad n=140\end{array}$ & $\begin{array}{l}<2 \text { episodes of distressing } \\
\text { symptoms before death, no. }(\%)^{*} \\
\qquad n=339\end{array}$ & $p$ value \\
\hline Age, yr, mean \pm SD & $74.8(13.4)$ & $73.6(17.1)$ & 0.40 \\
\hline Female sex & $76(54.3)$ & $155(45.6)$ & 0.09 \\
\hline \multicolumn{4}{|l|}{ Hospital service at time of death (top 10) } \\
\hline Intensive care & $15(10.7)$ & $111(32.7)$ & $<0.001$ \\
\hline General medicine & $49(35.0)$ & $87(25.6)$ & 0.05 \\
\hline Cardiology & $9(6.4)$ & $33(6.9)$ & 0.3 \\
\hline Oncology & $22(15.7)$ & $14(4.1)$ & $<0.001$ \\
\hline Neurology & $2(1.4)$ & $12(3.5)$ & 0.4 \\
\hline Malignant hematology & $6(4.3)$ & $6(1.8)$ & 0.1 \\
\hline General surgery & $5(3.6)$ & $7(2.1)$ & 0.3 \\
\hline Radiation oncology & $7(5.0)$ & $5(1.5)$ & 0.1 \\
\hline Family medicine & $2(1.4)$ & $8(2.4)$ & 0.7 \\
\hline Gynecologic oncology & $5(3.6)$ & $4(1.2)$ & 0.1 \\
\hline \multicolumn{4}{|l|}{ No. of inpatient encounters in the last $6 \mathrm{mo}$} \\
\hline 0 & $76(54.3)$ & $231(67.9)$ & 0.005 \\
\hline 1 & $36(25.7)$ & $63(18.5)$ & 0.08 \\
\hline 2 & $15(10.7)$ & $24(7.1)$ & 0.20 \\
\hline 3 & $5(3.6)$ & $17(5.0)$ & 0.6 \\
\hline$\geq 4$ & $8(5.7)$ & $5(1.5)$ & 0.007 \\
\hline Inpatient days in the last year, mean $\pm S D$ & $14.4 \pm 27.1$ & $10.1 \pm 24.1$ & 0.09 \\
\hline Patient has a family physician & $135(96.4)$ & 299 (87.9) & 0.04 \\
\hline LOS, median (IQR) & $17.6(31.6)$ & $13.2(19.8)$ & 0.05 \\
\hline Predicted mortality & $0.29(0.19)$ & $0.31(0.20)$ & 0.3 \\
\hline Elixhauser comorbidity score, mean \pm SD & $15.5 \pm 9.3$ & $11.2 \pm 8.5$ & $<0.001$ \\
\hline Q1 -3 to 5 & $27(19.3)$ & $103(30.5)$ & \\
\hline Q2 6 to 12 & $25(17.9)$ & $100(29.6)$ & \\
\hline Q3 12 to 19 & $39(27.9)$ & $83(24.6)$ & \\
\hline Q4 19 to 48 & $49(35.0)$ & $52(15.4)$ & \\
\hline \multicolumn{4}{|c|}{ Patient wishes for resuscitation documented within $24 \mathrm{hr}$ of admission } \\
\hline Not documented & $36(25.7)$ & $100(29.7)$ & 0.4 \\
\hline Full resuscitation including CPR & $27(19.3)$ & $71(20.9)$ & 0.7 \\
\hline Resuscitation but no CPR & $11(7.9)$ & $51(15.0)$ & 0.04 \\
\hline No resuscitation & $66(47.1)$ & $117(34.4)$ & 0.01 \\
\hline Family present in the last $24 \mathrm{hr}$ of life & $106(75.7)$ & $272(80.0)$ & 0.3 \\
\hline $\begin{array}{l}\text { Patient wishes for no resuscitation documented } \\
\text { in chart at time of death }\end{array}$ & $127(90.7)$ & $241(71.1)$ & $<0.001$ \\
\hline $\begin{array}{l}\text { Patient wishes for no resuscitation documented } \\
\leq 24 \mathrm{hr} \text { before death }\end{array}$ & $18(12.9)$ & $81(23.9)$ & $<0.001$ \\
\hline Palliative care consulted & $63(45.0)$ & $81(23.8)$ & $<0.05$ \\
\hline
\end{tabular}


People with symptoms before death had higher comorbidity burden, were more likely to have a family doctor, more likely to be admitted to medical oncology and more likely to have an order for no resuscitation written in their charts within 24 hours of admission.

Consistent with previous research, we found that many patients experience symptoms before death. ${ }^{20,27,28}$ In the SUPPORT study, which included hospital patients with lifethreatening diseases, $50 \%$ of surrogates reported that the patient had experienced moderate to severe pain during the final 3 days of life. ${ }^{28}$ In our study, only $21.5 \%$ had pain scores of 5 out of 10 or greater documented in the final 48 hours of life. Although not directly comparable, this result suggests an improvement. Another recent study of end-of-life symptoms among inpatients found that $22 \%-67 \%$ of patients experienced pain during their final admission to hospital. ${ }^{29}$ This is not directly comparable, but shows that our result is in keeping with published reports of symptoms at the end of life.

Although some patients may choose less aggressive treatment of symptoms in favour of being more lucid, patients

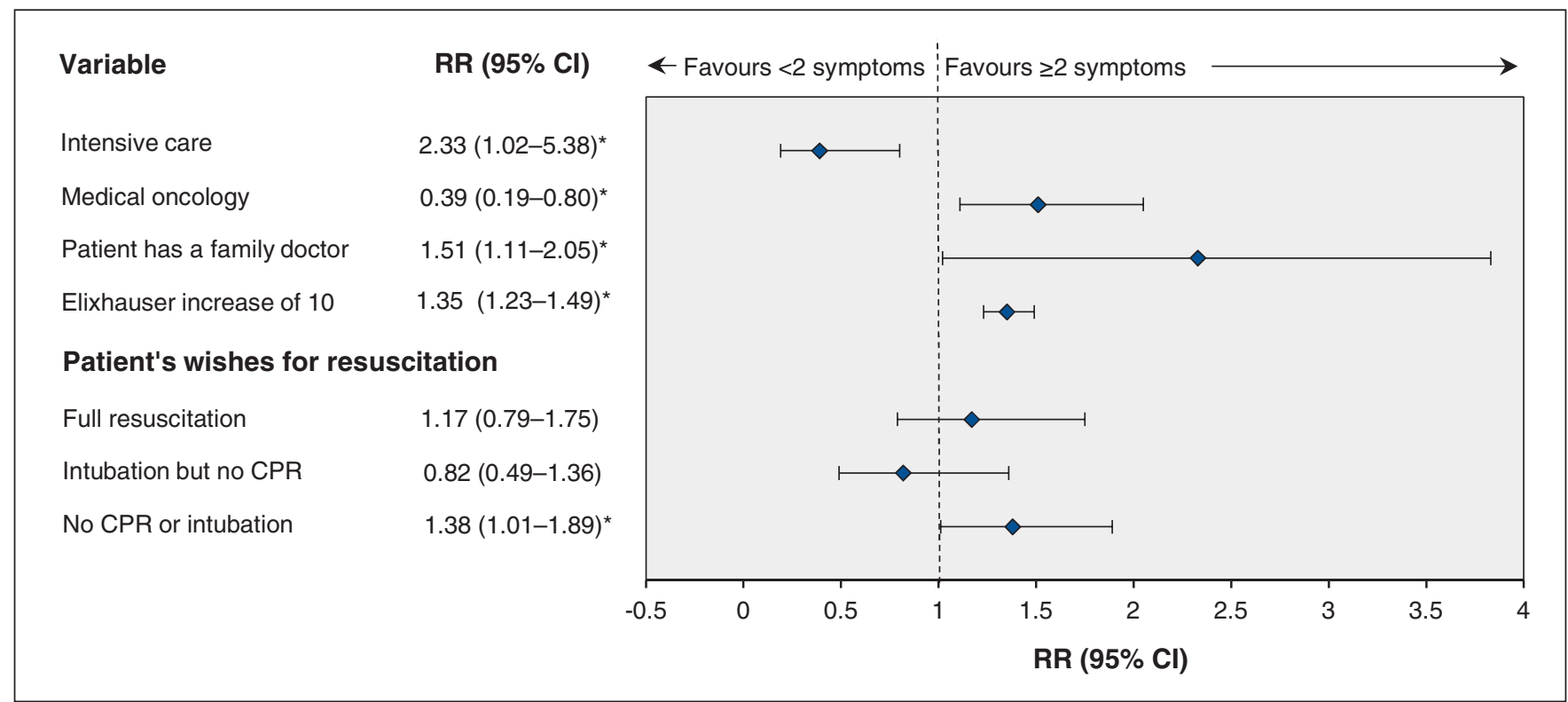

Figure 1: Patient- and encounter-level characteristics associated with presence of 2 or more symptoms in the last 48 hours of life. 'For patients' wishes for resuscitation as documented in the chart 24 hours after admission, the reference category was "not documented." $\mathrm{Cl}=\mathrm{confidence}$ interval, $\mathrm{CPR}=$ cardiopulmonary rescusitation, $\mathrm{RR}=$ risk ratio.

Table 4: Representative cases of patients who experienced multiple episodes of distressing symptoms in the final 48 hours of life

\begin{tabular}{|c|c|c|c|c|}
\hline \multicolumn{4}{|c|}{ Symptom } & \multirow[b]{2}{*}{ Case description } \\
\hline Pain & Dyspnea & Agitation & Nausea & \\
\hline 2 & 5 & 0 & 0 & $\begin{array}{l}\text { An 84-year-old man with metastatic renal cell carcinoma was admitted with delirium secondary to } \\
\text { hypercalcemia. He was given intravenous fluids. The patient began experiencing flank pain and } \\
\text { dyspnea. Initially, every recorded episode was treated with narcotics, but the following assessment } \\
\text { documents that the patient was still complaining of pain. The patient was getting frustrated with } \\
\text { the poor pain control. The patient had increasing dyspnea that was treated with benzodiazepines. } \\
\text { He died after } 3 \text { hours of increasing dyspnea. }\end{array}$ \\
\hline
\end{tabular}


need to be counselled about the typical frequency and severity of symptoms at the end of life so they can make educated choices. Patients consistently state that freedom from symptoms is one of the most important elements of quality end-oflife care. ${ }^{1,3}$ This is in contrast to the reality that many patients do experience symptoms at the end of life. If patients knew they were likely to experience symptoms at the end of life, some may choose more aggressive symptomatic treatment or physician-assisted death.

Comorbidity burden had the strongest association with symptoms before death in our study. This finding is likely because patients with multiple comorbidities experience more symptoms at all times, not just in the days before death. ${ }^{13,30,31}$ As other research has suggested, care for patients with multimorbidity is often inappropriately focused on diseasemodifying treatments instead of a palliative approach that prioritizes treatment of symptoms along with disease-modifying treatments. ${ }^{32-35}$ Further reinforcing the importance of a palliative approach, we found that patients with more symptoms at the end of life wanted limitations on the aggressiveness of care they received. Part of the challenge in implementing a palliative approach to care is the perception that palliative care is only for patients who have predictably terminal illnesses, such as incurable cancer. ${ }^{36,37}$ For patients with chronic progressive diseases with frequent exacerbations, a recovery from the current episode is often possible; therefore, focusing on prolonging life may seem appropriate. This is in contrast to the World Health Organization's model for palliative care that recommends therapy to alleviate suffering be started early in the disease course along with disease-modifying therapy. ${ }^{38}$

Patients who were admitted to medical oncology had more symptoms before death than those admitted to other specialties. This finding differs from previous studies that showed no difference in the symptom burden between patients who die from cancer compared with patients who die from other causes..$^{8,14,39}$ Our finding may be because only oncology patients with symptoms are admitted for end-of-life care, whereas patients with fewer symptoms remain at home.

We found an association between having a family doctor and more symptoms at the end of life. It is difficult to imagine a causal pathway for this association. Instead, the association could be because patients who have more symptoms and more severe disease are more likely to have a family physician.

Perhaps the most intriguing and concerning finding was that patients who explicitly ask not to be resuscitated are more likely to experience symptoms. This suggests that patients who are asking for limitations in the aggressiveness of care may not be getting the symptom-focused care they want.

Finally, we found that patients admitted to the ICU experience fewer symptoms before death. These patients are sedated and therefore unable to report symptoms. In addition, the 1-to-1 nursing ratio in ICUs may facilitate rapid assessment and treatment of any symptoms.

\section{Strengths and limitations}

Our study was single-centre and therefore may not be generalizable to other hospitals or countries. Nevertheless, our large mul- tifacility hospital provides a substantial proportion of the acute care health services in a large Canadian urban centre, and our findings are unlikely to be unique to our centre. Chart review is retrospective and observational, and differential measurement of the outcome on different wards may have introduced bias. Substantial effort at the Ottawa Hospital has been made to standardize hourly nursing assessments and documentation, which may mitigate some concerns over primary outcome assessment. We believe that, if anything, our outcome ascertainment method will underestimate the prevalence of symptoms because staff will not document symptoms that are not present but may, at times, miss symptoms. Our study did not evaluate whether symptoms were treated effectively, and therefore our results do not reflect on the quality of end-of-life care. Despite this weakness, our results are important because patients value symptom control at the end of life, and information about symptoms is important for both physicians and patients.

\section{Conclusion}

In a group of unselected patients who died in hospital, almost 1 in 3 experienced symptoms in the last 48 hours of life. The high incidence of symptoms in patients with high comorbidity, who have asked for less-aggressive care, suggests that further work is needed to integrate a palliative approach into chronic disease care. Future work should seek to understand symptoms experienced at the end of life by people who receive ideal care. Knowledge translation work is needed to determine how accurate expectations about end-of-life symptoms influence patients' decisions about palliative care and physician-assisted death.

\section{References}

1. Heyland DK, Dodek P, Rocker G, et al.; Canadian Researchers End-of-Life Network(CARENET). What matters most in end-of-life care: perceptions of seriously ill patients and their family members. CMA7 2006;174:627-33.

2. Singer PA, Martin DK, Kelner M. Quality end-of-life care: patients' perspectives. FAMA 1999;281:163-8

3. Steinhauser KE, Christakis NA, Clipp EC, et al. Factors considered important at the end of life by patients, family, physicians, and other care providers. 7AMA 2000;284:2476-82.

4. Health care use at the end of life in Western Canada. Ottawa: Canadian Institute for Health Information; 2007.

5. Health care use at the end of life in Atlantic Canada. Ottawa: Canadian Institute for Health Information; 2011.

6. Higginson IJ, Sen-Gupta GJ. Place of care in advanced cancer: a qualitative systematic literature review of patient preferences. 7 Palliat Med 2000;3:287-300.

7. Langford DJ, Tripathy D, Paul SM, et al. Trajectories of pain and analgesics in oncology outpatients with metastatic bone pain. 7 Pain 2011;12:495-507.

8. Solano JP, Gomes B, Higginson IJ. A comparison of symptom prevalence in far advanced cancer, AIDS, heart disease, chronic obstructive pulmonary disease and renal disease. 7 Pain Symptom Manage 2006;31:58-69.

9. McCarthy EP, Phillips RS, Zhong Z, et al. Dying with cancer: patients' function, symptoms, and care preferences as death approaches. 7 Am Geriatr Soc 2000; 48(Suppl):S110-21.

10. Mercadante S, Casuccio A, Fulfaro F. The course of symptom frequency and intensity in advanced cancer patients followed at home. 7 Pain Symptom Manage 2000;20:104-12.

11. Nordgren L, Sorensen S. Symptoms experienced in the last six months of life in patients with end-stage heart failure. Eur 7 Cardiovasc Nurs 2003;2:213-7.

12. Ellershaw J, Smith C, Overill S, et al. Care of the dying: setting standards for symptom control in the last 48 hours of life. 7 Pain Symptom Manage 2001;21:12-7.

13. Janssen DJ, Spruit MA, Wouters EF, et al. Daily symptom burden in end-stage chronic organ failure: a systematic review. Palliat Med 2008;22:938-48.

14. Tranmer JE, Heyland D, Dudgeon D, et al. Measuring the symptom experience of seriously ill cancer and noncancer hospitalized patients near the end of life with the memorial symptom assessment scale. 7 Pain Symptom Manage 2003; $25: 420-9$. 
15. Detering KM, Hancock AD, Reade MC, et al. The impact of advance care planning on end of life care in elderly patients: randomised controlled trial. BM7 2010;340:c1345.

16. Sinuff T, Dodek P, You JJ, et al. Improving end-of-life communication and decision making: the development of a conceptual framework and quality indicators. 7 Pain Symptom Manage 2015;49:1070-80.

17. Elixhauser A, Steiner C, Harris DR, et al. Comorbidity measures for use with administrative data. Med Care 1998;36:8-27.

18. van Walraven C, Austin PC, Jennings A, et al. A modification of the Elixhauser comorbidity measures into a point system for hospital death using administrative data. Med Care 2009;47:626-33.

19. Escobar GJ, Greene JD, Scheirer P, et al. Risk-adjusting hospital inpatient mortality using automated inpatient, outpatient, and laboratory databases. Med Care 2008;46:232-9.

20. Kehl KA, Kowalkowski JA. A systematic review of the prevalence of signs of impending death and symptoms in the last 2 weeks of life. Am 7 Hosp Palliat Care 2013;30:601-16.

21. Boonstra AM, Stewart RE, Koke AJ, et al. Cut-off points for mild, moderate, and severe pain on the numeric rating scale for pain in patients with chronic musculoskeletal pain: variability and influence of sex and catastrophizing. Front Psychol 2016;7:1466.

22. Hirschfeld G, Zernikow B. Variability of "optimal” cut points for mild, moderate, and severe pain: neglected problems when comparing groups. Pain 2013; 154:154-9.

23. Gerbershagen HJ, Rothaug J, Kalkman CJ, et al. Determination of moderateto-severe postoperative pain on the numeric rating scale: a cut-off point analysis applying four different methods. Br 7 Anaesth 2011;107:619-26.

24. Downey L, Curtis JR, Lafferty WE, et al. The Quality of Dying and Death Questionnaire (QODD): empirical domains and theoretical perspectives. 7 Pain Symptom Manage 2010;39:9-22.

25. McNutt LA, Wu C, Xue X, et al. Estimating the relative risk in cohort studies and clinical trials of common outcomes. Am F Epidemiol 2003;157:940-3.

26. Spiegelman D, Hertzmark E. Easy SAS calculations for risk or prevalence ratios and differences. Am 7 Epidemiol 2005;162:199-200.

27. Levin ML, Berry JI, Leiter J. Management of pain in terminally ill patients: physician reports of knowledge, attitudes, and behavior. 7 Pain Symptom Manage 1998;15:27-40.

28. A controlled trial to improve care for seriously ill hospitalized patients. The study to understand prognoses and preferences for outcomes and risks of treatments (SUPPORT). The SUPPORT Principal Investigators. $7 A M A$ 1995;274:1591-8.

29. Yao Y, Keenan G, Al-Masalha F, et al. Current state of pain care for hospitalized patients at end of life. Am 7 Hosp Palliat Care 2013;30:128-36.

30. Currow DC, Clark K, Kamal A, et al. The population burden of chronic symptoms that substantially predate the diagnosis of a life-limiting illness. $f$ Palliat Med 2015;18:480-5.

31. Rodriguez KL, Hanlon JT, Perera S, et al. A cross-sectional analysis of the prevalence of undertreatment of nonpain symptoms and factors associated with undertreatment in older nursing home hospice/palliative care patients. Am 7 Geriatr Pharmacother 2010;8:225-32.

32. Fitzsimons D, Mullan D, Wilson JS, et al. The challenge of patients' unmet palliative care needs in the final stages of chronic illness. Palliat Med 2007; 21:313-22.

33. Gore JM, Brophy CJ, Greenstone MA. How well do we care for patients with end stage chronic obstructive pulmonary disease (COPD)? A comparison of palliative care and quality of life in COPD and lung cancer. Thorax 2000; 55:1000-6.

34. Cleary J, Drennan J. Quality of life of patients on haemodialysis for end-stage renal disease. 7 Adv Nurs 2005;51:577-86.

35. Gibbs JS, McCoy AS, Gibbs LM, et al. Living with and dying from heart failure: the role of palliative care. Heart 2002;88(Suppl 2):ii36-9.

36. Bruera E. The development of a palliative care culture. 7 Palliat Care 2004; 20:316-9.

37. Hanks G. Palliative care: careless use of language undermines our identity. Palliat Med 2008;22:109-10.

38. National cancer control programmes - Policies and managerial guidelines. 2nd ed. Geneva: World Health Organization; 2002.

39. Saini T, Murtagh FE, Dupont PJ, et al. Comparative pilot study of symptoms and quality of life in cancer patients and patients with end stage renal disease. Palliat Med 2006;20:631-6.

Affiliations: Department of Medicine (Kobewka, Mulpuru, Forster), Division of General Internal Medicine, The Ottawa Hospital, University of Ottawa; Ottawa Hospital Research Institute (Kobewka, McIsaac, Mulpuru, Forster), Ottawa, Ont.; Department of Community Health Sciences (Ronksley), University of Calgary, Calgary, Alta.; Department of Anesthesiology and Pain Medicine (McIsaac) - The Ottawa Hospital, University of Ottawa; Performance Measurement (Forster), The Ottawa Hospital, Ottawa, Ont.

Contributors: Daniel Kobewka and Alan Forster conceived of the study, performed data collection and analysis, and wrote the manuscript. Paul Ronksley, Dan McIsaac and Sunita Mulpuru contributed to the analysis and interpretation of results. All of the authors critically revised the manuscript, approved the final version for publication and agreed to act as guarantors of the work.

Acknowledgements: This research was supported by Ministry of Research, Innovation and Science, and The Ottawa Hospital Academic Medical Organization.

Supplemental information: For reviewer comments and the original submission of this manuscript, please see www.cmajopen.ca/content/5/1/ E222/suppl/DC1 